\title{
THE LOW INTENSITY RESISTANCE TRAINING WITH BLOOD FLOW RESTRICTION IMPROVES MUSCLE STRENGTH, MUSCLE MASS AND PHYSICAL FUNCTION WITHOUT CHANGING THE DISEASE ACTIVITY IN RHEUMATOID ARTHRITIS PATIENTS
}

Leonardo Peterson dos Santos ${ }^{1, \star}$, Rafaela Cavalheiro do Espírito Santo ${ }^{2}$, Thiago Rozales Ramis ${ }^{1}$, Émerson Pena ${ }^{1}$, Vanessa

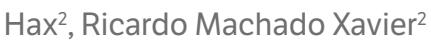

1.Universidade Federal do Rio Grande do Sul, Porto Alegre (RS), Brazil; 2.Hospital de Clínicas de Porto Alegre, Porto Alegre (RS), Brazil. *Corresponding author: leonardosantos@hcpa.edu.br

\section{BACKGROUND}

Rheumatoid arthritis (RA) patients present changes in physical function and body composition. Studies have been demonstrated that the high intensity resistance training (HIRT) is a tool able to improve these parameters in RA patients. However, some RA patients do not adhere to HIRT. Thus, understanding the effects of low intensity training with blood flow restriction (TBFR) as a training alternative is fundamental for this population. This study aims to assess the efficacy of TBFR on muscle strength, muscle mass and physical function in RA patients.

\section{METHODS}

Eighteen women (planned, $n=45$ ) with RA were randomized into TBFR or HIRT. The training program had duration of twelve weeks. The exercises performed were knee extension, dorsal pull and biceps curl. Muscle strength was assessed by 1 maximum repetition (1RM) and handgrip. Muscle mass was assessed by ultrasound (Esaote). Physical function was assessed by time-up and -go (TUG) and $30 \mathrm{~s}$ sit to stand (STS) tests. Disease activity was assessed by DAS-28. The student's t test for independent samples and the pairwise student test were performed; $p<0.05$ was considered as a significant difference.

\section{RESULTS}

Eleven women completed the study before the study had to be interrupted because of the COVID-19 pandemic (mean age: TBFR $=57.14 \pm 6.67$ years $=$ HIRT, $61.00 \pm 2.00$ years; $p=0.30$ ). The majority patients showed mild disease activity at baseline ( TBFR $=2.91 \pm 0.89 ; \mathrm{HIRT}=2.87 \pm 1.72, p=0.95$ ) and no significant statistically difference after training protocol were found (TBFR, $p=0.36 ; H I R T, p=0.69)$. The musclestrength of knee extension increased after training program on TBFR $(+3.29 \pm 2.50 \mathrm{~kg}, \mathrm{p}=0.01)$ and HIRT $(+5.13 \pm 1.11 \mathrm{~kg}, p=0.003)$; however, no significant statistically difference was found between groups $(p=0.20)$. The muscle strength of dorsal pull test, which does not involve blood restriction, increased only in HIRT $(+6.50 \pm 2.08 \mathrm{~kg}, p=0.008)$. On the other hand, only TBFR was able to improve the right handgrip strength $(+6.71 \pm 4.61 \mathrm{~kg}, p=0.008)$ and left handgrip strength $(+7.14 \pm 6.04 \mathrm{~kg}, p=0.02)$, showing a statistically significant difference between groups $(p<0.05)$. The muscle mass increased after training program in the quadriceps musculature for TBFR (1.17 $\pm 0.58 \mathrm{~cm}, p=0.005)$, but not for HIRT $(0.79 \pm 0.75 \mathrm{~cm}, p=0.21)$, however there was no significant statistically difference between groups $(p=0.43$ ). Muscle strength and biceps muscle mass did not increase in both interventions after the training program. The function evaluated by the TUG test showed improvements in runtime for TBFR $(-0.70 \pm 0.68 \mathrm{~s}, p=0.03)$, but not for HIRT $(-0.19 \pm 0.79 \mathrm{~s}$, $p=0.67)$, however also without difference between groups $(p=0.28)$. No statistically significant difference was found after training program in TBFR or HIRT for STS test.

\section{CONCLUSION}

These preliminary observations indicate that TBFR can improve muscle strength, muscle mass and physical function without changing the disease activity in RA patients.

\section{KEYWORDS}

Rheumatoid arthritis, Kaatsu training, Vascular occlusion. 